\title{
la mayor fábrica de cemento del munalo
}

En el número 95 de esta Revista (reptiembre-octubre de 1959) dábamos una breve noticia sobre esta factoría que se está construyendo en Atchinisk (Siberia Central). En este mismo sentido escueto se ha hecho referencia en otras publicaciones, nacionales y extranjeras, a esta gran instalación solicitada a una firma francesa por el Gobierno de la U. R. S. S., destacando las propiedades más características, tales como producción, dimensiones de hornos y molinos, sistemas de fabricación, etc.

En este artículo, cedido amablemente por Tecnicom, S. A., vamos a tratar, con todo detalle, de todas y cada una de las Secciones que componen esta fábrica.

Se dice que es la mayor del mundo. Esto es absolutamente exacto. Su producción será de 5.700 toneladas de cemento por día. La diferencia con las más grandes construídas hasta la fecha es de 1.700 toneladas. En efecto, las que actualmente existen en Bélgica y en Estados Unidos no rebasan las 4.000 toneladas de producción al día; esto, a pesar de que se trata de fábricas construidas hace ya mucho tiempo y que han llegado a estas cifras en etapas sucesivas.

Las materias primas que alimentarán esta fábrica serán la caliza y la nefelina (subproducto de la fabricación del aluminio).

E1 procedimiento a seguir es el de vía húmeda en su totalidad, con $32 \%$ de agua en la pasta.

\section{disposición general de la fábrica}

No pertenece a las de forma clásica, cuyo horno está emplazado perpendicular o paralelamente a la nave de almacenamiento. Su disposición, por el contrario, es de una doble $\mathrm{T}$.

A esta disposición se llegó debido a que ha sido necesario prever dos naves de almacenamiento, una en cada extremo, por el gran tonelaje de materias crudas, carbón, nefelina y yeso, dando como resultado el que, desde la llegada de la caliza hasta la expedición del cemento, hay 785 metros de dístancia.

La superficie total de la fábrica propiamente dicha cubre 16 hectáreas.

De acuerdo con la disposición general del plano de emplazamiento (fig. 1), esta fábrica se divide en seis secciones principales, que llamaremes $a, b, c, d, e$ y $f$.

\section{sección a}

\section{mezcla de las materias primas y vasos de pasta}

En esta Sección figuran la llegada de materias primas, la trituración y mezclado y los vasos de pasta.

La caliza llega a la fábrica ya machacada y se vierte, mediante vagones-tolva, directamente a la nave central de almacenamiento. En esta nave trabajan dos puentes-grúa, con cucharas de $7 \mathrm{~m}^{3}$. Estos puentes tienen $31,5 \mathrm{~m}$ de luz, con una carga unitaria de $25 \mathrm{t}$. La potencia motriz de cada puente es de dos motores de $100 \mathrm{CV}$, un motor de $40 \mathrm{CV}$ y otro de $150 \mathrm{CV}$

La caliza se vierte mediante los puentes-grúa en cinco tolvas, las cuales, a su vez, alimentan cinco molinos. Estos molinos son tubulares de $3,200 \times 14 \mathrm{~m}$, con tres compartimentos y un peso unitario de $260 \mathrm{t}$. Blindajes con placas de acero al manganeso. Cuerpos moledores y cilpebs con un peso de $110 \mathrm{t}$. Sistema de ataque lateral con motores asíncronos sincronizados de $2.500 \mathrm{CV}$ a $3.000 \mathrm{~V}$. Producción: 75 t/hora en seco.

Los reductores y los carters de los molinos han sido equipados con sistemas de calefacción a vapor o agua caliente y de refrigeración con agua fría, debido a las diferencias de temperaturas en que trabajará la maquinaria (en invierno $-40^{\circ} \mathrm{C}$; en verano $+35^{\circ} \mathrm{C}$ ). La circulación del agua o vapor se realiza en tuberías de cobre.

Este gran problema de las temperaturas exteriores ha hecho que todas las instalaciones hayan sido dotadas de calefacción y estén situadas bajo techado. 
La entrada en los mismos molinos del lodo de nefelina se realiza mediante bombas rotativas. Los ensayos de laboratorio se han efectuado con pastas de $28 \%$ de agua; pero el trabajo se realizará con pastas con un 35 c de agua, tomándose precauciones necesarias para evitar la decantación de la nefelina, por su poca plasticldad.

Las pastas se dosifican por medio de dosificadoras-pesadoras automáticas, controladas y registradas instantáneamente desde una cabina central, desde la cual, al mismo tiempo y por medio de este sistema, son corregidas en la proporción debida.

A la salida de los molinos, las pastas son enviadas mediante bombas rotatívas con acoplamiento por manguito elástico y dírecto con aletas de caucho, a una batería de silos de hormigón armado. Para evitar la decantación en eștos silos, existe un sistema de soplado con aire comprimido por el fondo y los lados,

Estas bombas de envio de pastas a silos tienen una capacidad umitaria de 300 m3/hora y han sido previstas para pastas con un $27 \%$ de agua, pesando de 1,7 a $2,07 \mathrm{~kg} / \mathrm{dm}$.

Doce cubas de mezcla de $10 \mathrm{~m}$ de diśmetro y $19 \mathrm{~m}$ de altura, con una capacidad unitaria de $2.400 t$, continúan las Instalaciones descritas.

Para asegurar el transvase entre si y el envio a los tanques planetarios de $25 \mathrm{~m}$ de diámetro y una capacfdad unitaria de $2.500 \mathrm{~m}^{3}$, se han previsto cuatro bombas rotativas de $300 \mathrm{~m} / \mathrm{h}$, iguales a las descritas anteriormente.

Los tanques de agitación de pasta trabajan lenta y continuamente por medio de seis agitadores rotativos accionados cada uno por moto-reductores de 8,5 CV de potencia.

\section{Stererido b}

\section{cocçón del clínker}

Según hemos señalado anteriormente, el elínker se cuece en dos hornos rotativos de $175 \mathrm{~m}$ de longitud y están constituídos por 20 virolas que serón ensambladas a pie de fábíca por soldadura eléctrica. Las dimensiones y el peso de estas virolas va desde $\emptyset 6,118$ y 6 m de longitud, con un peso de 83,700 t, a $\varnothing 5,098$ con 10,700 m de longitud, con un peso de 66 toneladas.

En cada horno (fig. 2) cabe distinguir:

1. La zona de secado, con un $\emptyset$ de $5,800 \mathrm{~m}$, repestida con ladrillos de desgaste de elevado contenido en sílice y cadenas intercambiadoras de calor con un peso de $250 \mathrm{t}$ por horno.

2. La zona de descarbonatación, con un $\varnothing \mathrm{dz} 4,800 \mathrm{~m}$, revestida con ladriLlos aislantes.

3. La zona de coccion, con un $\varnothing$ de $5,300 \mathrm{~m}$, revestida con Iadrillos de magnesia.

El peso total de cada horno en orden de marcha es de $1.325 \mathrm{t}, \mathrm{y}$ se apoya mediante siete caminos de rodaduras, con anchos diferentes.

También en estos hornos se ha empleado un procedimiento totalmente nuevo para conseguir que el tubo se mantenga exactamente en su posición longitudinal. El sistema que se ha seguido es mediante cinco roldanas de empuje axial de reglafe hidráulico, las cuales evitan el empuje longitudinal. Hasta el presente, este empuje longitudinal se compensaba generalmente sesgando las roldanas. Así se consigue que el movimiento axial de la roldana, montada sobre rodamientos, gire en un plano horłzontal, conservando los caminos de rodadura del tubo constantemente en planos perfectamente lisos.

La velocidad de rotación del horno está comprendida entre 0,6 y 1,2 r.p.m. Para ello, está equipado con dos motores de $450 \mathrm{CV}$, asincronos y mando con reostato de deslizamiento y reductor de siete vueltas.

\section{filtro de humos}

Electrostático, con hilos de acero $\mathrm{y}$ chapas verticales goipeadas con martillos vibradores.

\section{enfricdores}

Del sistema Fuller con parrilla inclinada. Cada horno va equipado con un enfriador de 4,270 $\times 22,860 \mathrm{~m}$ y $290 \mathrm{t}$ de peso. El rendimiento con que han sido garantizados es de $2.500 \mathrm{t}$ de clínker por día, para cada horno, con un enfriamiento fuerte. El enfriamiento se produce por una caida rápida de temperatura de $1.370^{\circ} \mathrm{C}$ a $65^{\circ} \mathrm{C}$. $\mathrm{Al}$ mismo tiempo, los enfriadores recirculan aire caliente.

Los enfriadores han sido dímensionados de forma que permiten emplear dos cadenas de evacuación de $400 \mathrm{~mm}$ de ancho. A la salida del enfriador se han situado, alimentadas por cada una de las cadenas, dos machacadoras de clínker por aparato.

\section{evacuacjón del cllaker}

El sistema empleado ha sido concebido de tal forma, que con sólo el funcionamiento de dos cadenas de 500 mm de ancho de las cuatro instaladas, sea posible, con interconexiones, evacuar la totalidad de la producción de los dos hornos. La capacidad de cada una de las cadenas es 104 t/hora, es decir, la producción total de cada horno. Cuatro básculas registradoras permitirán conocer el peso total de clinker por cada cadena.

El clinker enfriado cae directamente en la nave de almacenamiento, en donde es recogido por uno de los tres puentes-grúa con cuchara de $7 \mathrm{~m}:$ descritos anteriormente. 
CONSERMACION, ENSACADO FICACON DE CAREON

MEZOLA DE MATERIAS PRMMAS Y VASOS DE PASTA DEPARTAMENTO DE COCCKON
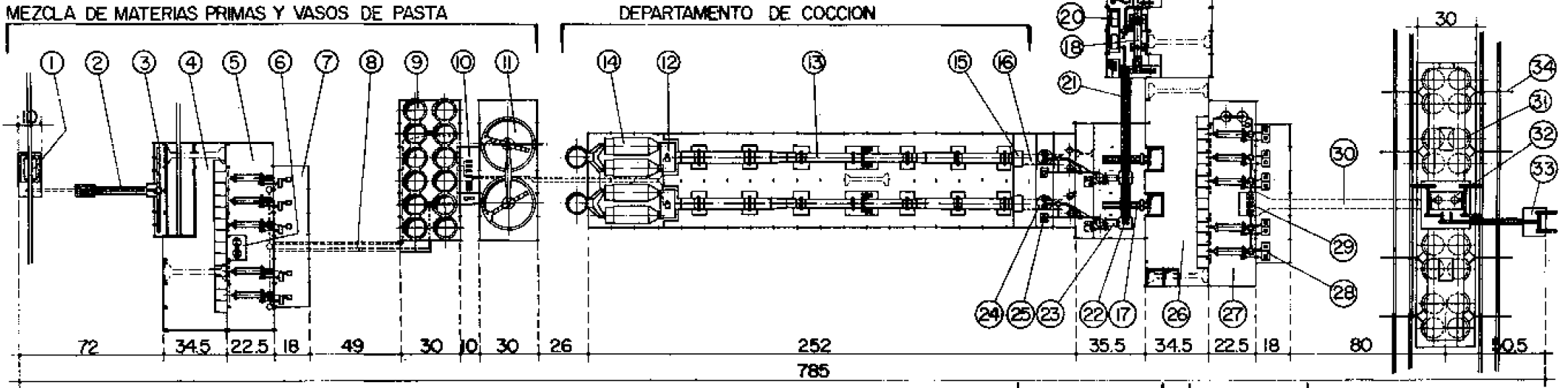

Fis: 1

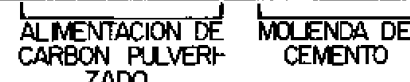

sección_d

sección e

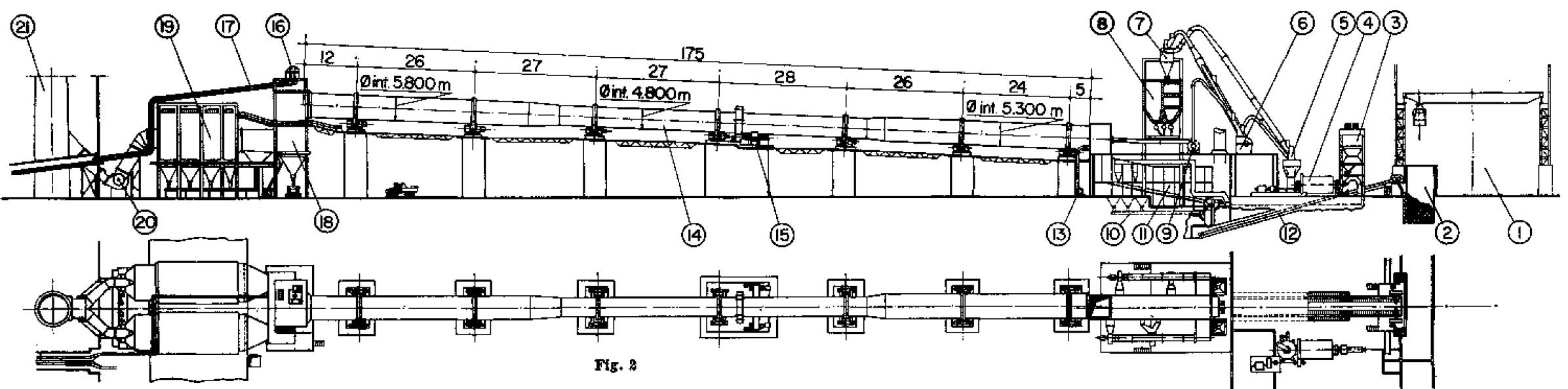




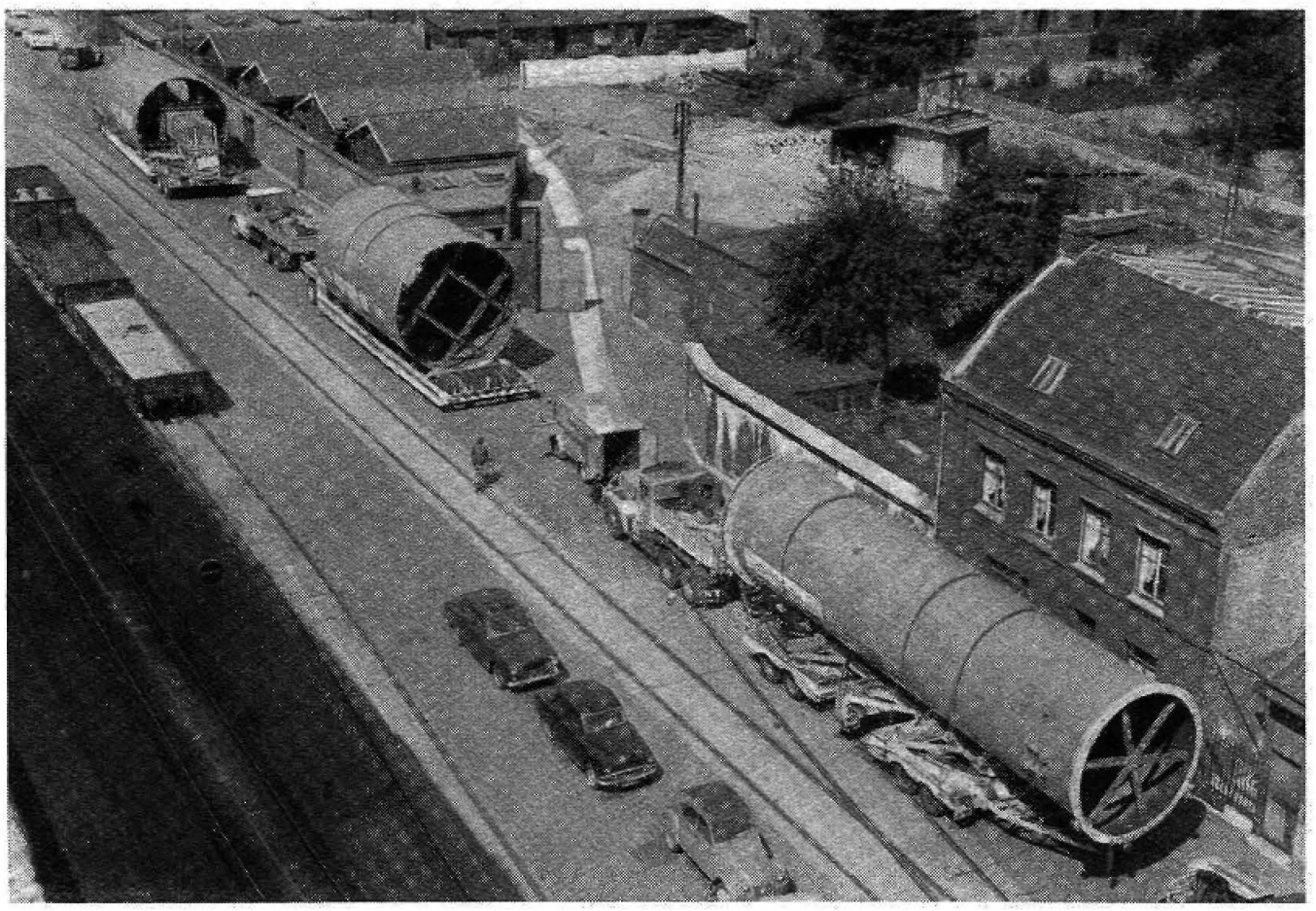

Fig. 3

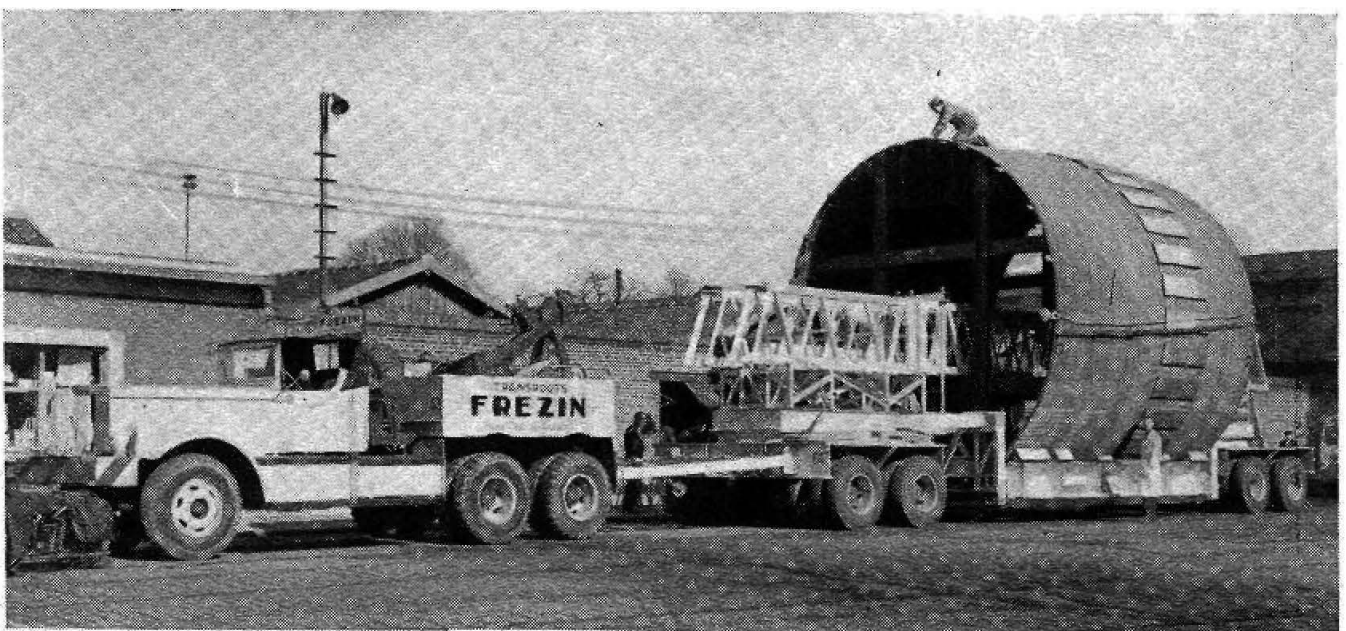

Fig. 4

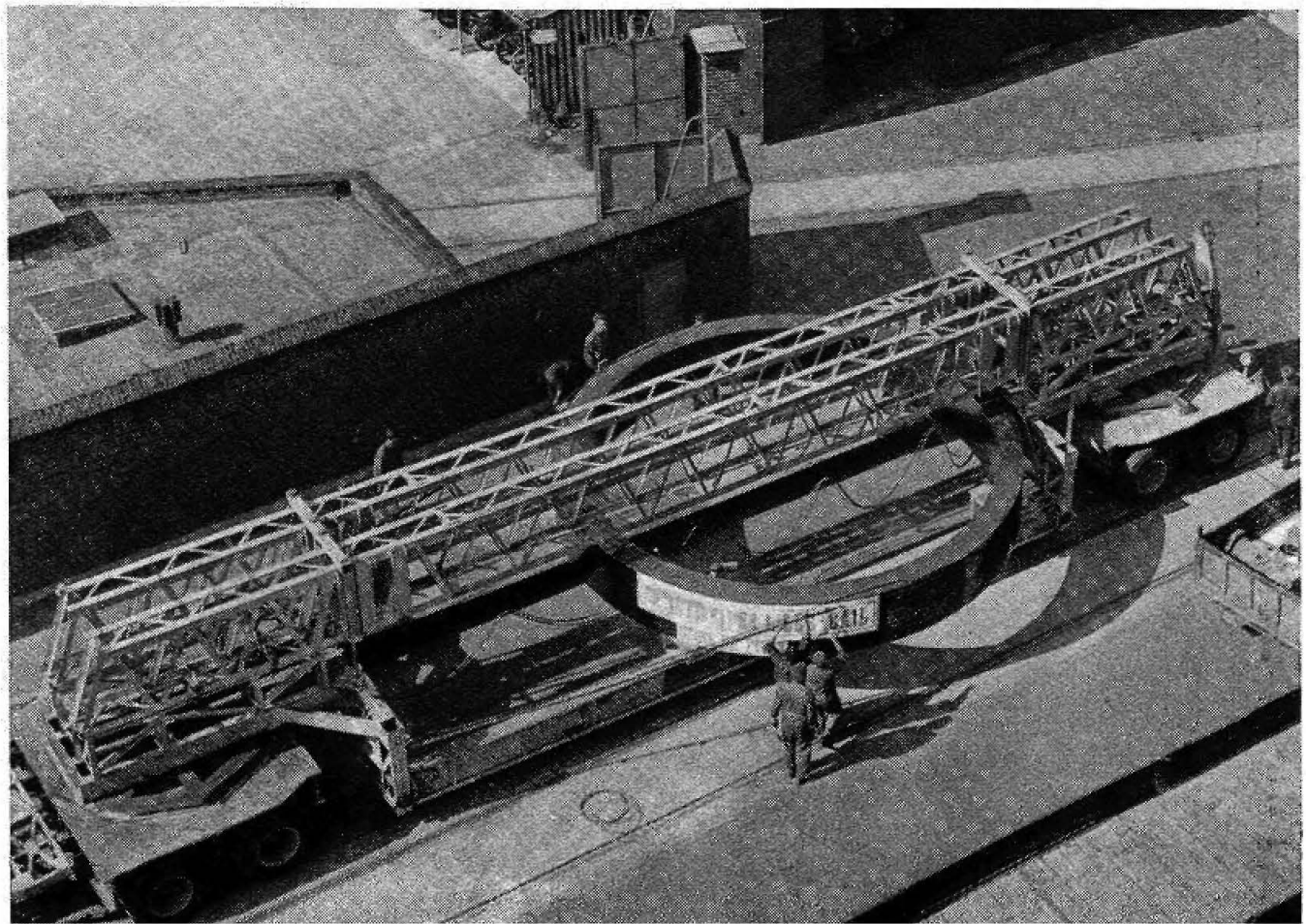

Fig. 5 


\section{seretín c}

\section{almacenamiento, distribución, secado y dosificoción del carbón}

Los hornos funcionarán con lignitos de Siberia del Ncrte, mezclados con menudos de llama corta. El consumo de combustible será de $25 \mathrm{t} /$ hora, con un poder calorifico de 5.900 calorias. El combustible se almacenará en el interior de la nave central por medio de los puentes-grúa.

El secado de los lignitos se realizaré con un secador rotativo da $3,600 \mathrm{~m}$ de diámetro y $25 \mathrm{~m}$ de longit d, accionado por un motor de $100 \mathrm{CV}$ y calentado con un quemedor de carbón pulverizado, utilizando el mismo combustible que el horno, con una bomba de 5 pulgadas (12,5 centímetros).

Una vez seco el lignito, conteniendo de 0,05 a $1 \%$ de agua, serf conducldo a las tolvas de mezcla, por medio de cintas transportadoras. Los menudos antracitosos pasarán directamente a las tolvas por no ser necesario el secado previo.

Desde las tolvas y valiéndose de los mismos distribuidores-pesadores que se utilizán para las materias primas, la mezcla pasa a un molino ventilado de $3,200 \mathrm{~m}$ de diámetro y $6,500 \mathrm{~m}$ de longitud, con una capazidad de molienda de 32 t/hora, para ser pulverizada. Fl aire caliente que entra en este molino proceda del enfriamiento del clínker. Para la recuperación del polvo se instalarán captadores electrostáticos.

\section{secrion d}

\section{alimentación del horno con carbón pulverizado}

El carbón pulverfzado en el molino descrito en el apartado anterior, 'se acumula en una tolva. Desde esa tolva, y con ayuda ds un alimentador de aletas rotativas, pasa al quemador, que es del tipo telescopico con servo-motores y calentamien ío previo. El polvo recogido del cajtador del horno, se introduce en el quemador al mismo tiempo que el combistible, en la debida proporción $\mathrm{y}$ de acuerdo con el contenido en Álcalis.

La tobera del horno, por medio del sistema telescópico, se desplazará en todas direcciones, permitiencio, además, que gire constantemente sobre su eje, evitando los desgastes locales prematuros y que se curve, con Io ctal dafiaria los ladrillos refractarlos.

\section{seceión $\mathrm{B}$}

\section{molienda del cemento}

La nave de molienda de cemento está dotada con cinco molinos, cuatro de ellos para cemento Portland ordinario, con las mismas dimensiones y pesos que los descritos en el apartado a), pero trabajando en circuito abierto. Los cuerpos molturadores tendrán un peso de $170 \mathrm{t}$, en lugar de las $110 \mathrm{t}$. El quinto molino trabajará en circulto cerrado y producirá supercemento. Esta sala está considerada también la más grande del mundo, dada su capacidad de molturacjón, puesto que será capaz de producir 240 t/hora de cemento fino a 2.800 Blaines y 45 t/hora de cemento extrafino a 3.800 Blaines.

Los motores que accionan estos molinos serán asincronos sincronizados, de 2.500 CV y $3.000 \mathrm{~V}$, acoplados a reductores de la misma potencia y que trabajarán a 19 r.p.m. La alimentación de los molinos se hará con tres distribuidores-pesadores de $60,10 \mathrm{y} 3 \mathrm{t} /$ hora. Han sido previstos también depuradores electrostáticos, que, además de captar los polvos de los molinos, limpiarán la sala de molienda del polyo ambiental.

El problema de la clasificación de los cuerpos moledores desgastados por el uso, se resolverá utilizando un tobogán perforado, del mismo sistema que se usa para clasificar las naranjas.

La manipulación de estos cuerpos molturadores se efcctía por carretillas transportadoras-elevadoras y con ellas se cargan los toboganes o los molinos.

Esta disposición tan perfecta de concepción y estilo hará que sea fácil el problema de la carga y separación, y despejará en absoluto la nave.

El cemento es conducido por aero-deslizadores a un gran foso, desde el cual es bombeado, por msdio de szis bombas (una en reserva) de 10 pulgadas $(25,5 \mathrm{~cm})$ y con una capacidad de $100 \mathrm{t}$, a los silos de almasenamiento.

\section{geccibn}

\section{conservación del cemento ensacado y carga}

Dleciséls sllos de $11,500 \mathrm{~m}$ de diǵmetro y $30 \mathrm{~m}$ de altra, con una capacidad de 64.000 t de cemento, forman el equipo de almacenamiento de la fábrica. Estos silos están conectados entre si y, para ello, al sistema se le ha equipado de 100 válvulas servo-motores, Ias cualıs permitirán todas las combinaciones posibles. 
Para evitar la abrasión producida en los codos de las tuberias se han revestido estos codos de porcelana, con lo cual se facilita, además, el deslizamiento. También se han previsto dos sistemas de captación de polvos con mangas, que irán montadas en la parte superior de la baterfa de sllos.

Desde los stlos, el cemento cae por aero-deslizadores de tipo abierto, colocados en espiga, a dos aero-deslizadores principales de una longitud de $100 \mathrm{~m}$, los cuales están enlazados, a su vez, con Ia tubería principal. Por esta red principal el cemento cae a un toso, desde el cual, por medio de dos bombas de 8 pulgadas (20 cm), pasa directamente a las tolvas de las ensacadoras.

Las ensacadoras son de tipo rotativo $\mathrm{y}$ tienen una capacidad unitaria de 100 toneladas/hora.

La casi totalidad del cemento se cargará a granel sobre vagones, atilizando ocho lineas de aero-deslizadores, con accionamiento por motor. El sistema permite la carga simultánea de ocho vagones situados en líneas diferentes (debido a las condiciones atmosferticas reinantes en el territorlo donde está situada la fábrica, sólo se ha previsto una linea de carga de camiones).

\section{material de control, de regulación y de telemando}

Todas las Secciones enumeradas anteriormente están dotadas de accesorios, que realizarán todas las funciones de control, medida, accionamtento a distancia y regulación.

No obstante la complejidad de las Secciones de la fábrica y sus ajferentes necesidades, se ha logrado centralizar todas las funciones de los equipos automáticos y de control en solo diez cuadros, desde lọs cuales se acciona la totalidad de la fábrica.

La construcclón de esta fabrica gigantesca, la naturaleza y el tonelaje de las materias primas a tratar, los tonelajes de clínker a obtener, asi como todas las instalaciones, han planteacio problemas que se han resuelto de una manera elegante y concisa, evitando complejidades y sistemas de diffeil funcionamiento y entretenimiento.

La prueba mas concluyente nos la da el que, no obstante las características de su emplazamiento, sus gigantescas instalaciones y las manipulaciones que se han de hacer, esta fábrica permitirá obtener un rendimiento medio que se calcula en $1.600 \mathrm{t}$ de cemento por hombre $\mathrm{y}$ año.

Como dato curioso, y que da tambtén una ldea aproximada de la magnitud de esta fábrica, seŕalamos que, con sus $40.000 \mathrm{~kW}$ de potencia instalada, consumiré la misma cantidad de energia eléctrica que una citudad de 100.000 habitantes.

Para llegar al momento de la puesta en marcha, fue neoesario solucionar antes dos problemas esenciales: el de la fabricación de las piezas y el de su transporte hasta Siberia.

\section{problema metalúrgico}

Como es fácil suponer, este problema no ha sldo sencitlo. Necesito y planteó estudios de gran amplitud, diflcul. tades técnicas de toda clase que hubo que superar (caso de las plezas pesadas) y la puesta en obra de medios muy importantes.

Fue necesario: coler, soldar y mecanizar llantas de $400 \mathrm{~mm}$ de espesor con diámetros de 6,900 m; constituir virolas para los homos, precisando curvar $y$ soldar chapas de hasta $120 \mathrm{~mm}$ de espesor; controlar estas piezas con ei sistema clastco de los rayos $x$ y añadir a este procedimiento los ultrasonidos $y$ la bomba de cobalto, con objeto de poder garantizar a las plezas o a los trabajos efectuados una calidad rigurosa.

\section{problema del transporte}

Como punto final a todos los problemas resueltos, quedaba el último: el del transporte. Según los datos que se conocian de la región, solamente se podía hacer llegar la maquinaria durante el periodo de congelación de los rios, para que éstos pudleran servir de pista.

Desde la fábrica, situada al norte de Francia, se envaron en remolques especiales las clncuenta y cuatro plezas que constituian el grueso de la expedición; algunas de estas piezas pesaban $60 \mathrm{t}$, otras $30 \mathrm{t}$. En total, se enviaron $14.000 \mathrm{t}$ (figs. 3,4 y 5 ).

E1 transporte se efectuó de noche por carreteras mimuclosamente estudiadas. Para paser por Brtaselas, hacia Amberes fue necesario, en alguna calle, levantar los cables del tendido electrico de los tranvias. Desde este puerto se utilizs la vía marítima hasta Iguarka, en la desembocadura del río Yenisei. A continuación, en barcazas, a lo largo del rio, durante los meses de julio y agosto, unicos meses que es navegable.

Después de ser descargada la maquinaria en Krasnolarsr, fueron a Atchinsk, lugar de emplazamlento de la ta. brica, medlante trineos, esperando para ello que los rios que habia que atravesar estuvieran helados.

Las piezas de menos importancia se transportaron por ferrocarril hasta Atchinsk, lugar por donde pasa el Transibertano. 